\title{
BUCHBESPRECHUNGEN
}

\section{Fontes Historiae Juris Gentium. Quellen zur Geschichte des Völkerrechts}

Hrsg. von Wilhelm G. Grewe, in Zusammenarbeit mit dem Institut für Internationales Recht an der Freien Universität Berlin, Band 2, 1493-1815

Berlin und New York: de Gruyter, 741 S., DM 368,-

Die von Grewe herausgegebene Sammlung von Dokumenten zur Geschichte des Völkerrechts schließt eine Lücke. Es existiert weder in deutscher noch in irgendeiner anderen Sprache ein vergleichbares Quellenwerk. Zwar erfaßt die von Clive Perry herausgegebene "The consolidated treaties series" mit ihren über 230 Bänden nahezu alle internationalen Staatsverträge, einschließlich der sogenannten Kolonialverträge seit 1648 . Jedoch beschränkt sich dieses Werk auf die Verträge und enthält keine Dokumente anderer Art. Dagegen gibt die von Grewe herausgegebene Sammlung auch wichtige andere Dokumente, wie etwa Repressalien- und Kaperbriefe, Gesetze, Proklamationen, päpstliche Bullen und nationale Prisenordnungen wieder. Dabei ist das Werk auf drei Bände angelegt. Der nunmehr aus praktischen und technischen Gründen als erster erschienene Band 2 erfaßt die Periode von 1494 bis 1815. Der dritte Band wird die Periode von 1815 bis 1945 zum Gegenstand haben und Band 1 die älteren Perioden von der Antike bis zum Ausgang des Mittelalters umspannen.

Dabei folgt der Aufbau des Werkes den "Epochen der Völkerrechtsgeschichte", die der Herausgeber 1983 veröffentlicht hat. Die ungeheure Fülle des Stoffes macht schon das monumentale Quellenwerk "The consolidated treaties series" deutlich, das sich auf die internationalen Staatsverträge seit 1648 beschränkt und trotz dieser Beschränkung 230 Bände umfaßt. Diese Stoffülle zwingt den Herausgeber zu einer Auswahl der vorhandenen Quellen. So muß in der Regel auch auf den vollständigen Abdruck der jeweiligen Texte verzichtet werden, und es werden jeweils nur die Teile des Textes, die der Herausgeber für die wichtigsten hält, in der Auswahl wiedergegeben. Dabei erfolgt die Wiedergabe der Texte in der authentischen Originalsprache sowie einer deutschen und einer englischen Übersetzung.

Der Teil A des Bandes 2 hat die Periode von 1493 bis 1648 zum Gegenstand, die vom Verfasser im Anschluß an seine "Epochen der Völkerrechtsgeschichte" als spanisches Zeitalter bezeichnet wird. Dabei ist besonders zu begrüßen, daß Grewe neben den wichtigen spanisch-französischen Friedensverträgen bzw. den englisch-spanischen Friedensverträgen zwei wichtige Kolonialverträge zwischen den ostindischen Herrschern und Portugal bzw. Holland in die Sammlung aufgenommen hat. Auch die Proklamation der spanischen Konquistadoren bei ihren überseeischen Landnahmen in Amerika wird in der Sammlung wiedergegeben. Ebenso sind die wichtigsten Dokumente zur spanisch-portugiesischen 
Teilung der Neuen Welt, wie die Bulle des Papstes Alexander VI. vom 4. Mai 1493 und der spanisch-portugiesische Vertrag von Tordesillas vom 7. Juni 1494 in die Sammlung aufgenommen. Dokumente zu Krieg und Frieden jenseits der "Linie", zum Seekriegsrecht und zur Freiheit der Meere und zu den Handelskompanien und überseeischen Siedlungen runden den Teil $\mathrm{A}$ ab.

Der Teil B umfaßt die Zeit von 1648 bis 1815, die von Grewe im Anschluß an seine "Epochen der Völkerrechtsgeschichte" als französisches Zeitalter bezeichnet wird. Zurecht erscheinen zu Beginn des Abschnitts I, der die großen, die politische Ordnung Europas konstituierenden Friedensschlüsse zum Gegenstand hat, die beiden westfälischen Friedensverträge vom 14. bzw. 24. Oktober 1648. Auch die Verträge zum Frieden von Nimwegen 1678/79, zum Frieden von Ryseijk 1697, zum Frieden von Utrecht und Rastatt 1713/14, zum Frieden von Aachen 1748, zum Frieden von Paris 1763, zum Frieden von Versailles und Paris 1783/84 und zum Ersten Pariser Frieden sind in diesem Abschnitt enthalten. Abschnitt II gibt wichtige bilaterale und regionale Friedensverträge von gesamteuropäischer Tragweite wider. In Abschnitt III sind die wichtigen Barrière-Verträge von 1709 bzw. 1715 als regionale Sicherheitsverträge wiedergegeben. Teilweise nicht sehr geglückt scheint mir die Auswahl der Verträge zwischen Europa und den nicht-christlichen Mächten in Abschnitt IV. Dies betrifft vor allen den wiedergegebenen "Unterwerfungsvertrag" der östlichen Indianer mit dem Gouverneur von Massachusetts vom 11. August 1693 und den Friedens-, Freundschafts-, und Bündnisartikel des Superintendenten der britischen Krone mit den Huronen vom 18. Juli 1764. Diese Verträge, vor allem der erstgenannte, sind für die zwischen den europäischen Mächten bzw. den USA seit 1778 auf der einen Seite und den "Indian Nations" bzw. "Indian Tribes" üblicherweise geschlossenen Verträge untypisch. Gerade die britische Krone und später die USA haben mit den "Indian Nations" bzw. "Indian Tribes" Verträge auf der Basis der Gleichordnung abgeschlossen, die sich in Form und Inhalt kaum von völkerrechtlichen Verträgen zwischen europäischen Mächten in dieser Zeit unterscheiden. Dies wird durch die Wiedergabe der beiden eher untypischen Verträge nicht deutlich, die eher die unzutreffende These von Grewe in seinen "Epochen der Völkerrechtsgeschichte" zu stützen vermögen, daß es zwischen dem indianischen Gemeinwesen und den europäischen Mächten bzw. den USA keine völkerrechtlichen Verträge gab. Im Abschnitt V sind wichtige Dokumente zu vom Ius Publicum Europaeum garantierten Thronfolgeordnungen intemationaler Tragweite wiedergegeben. Zurecht sind in diesem Abschnitt die Verträge zur Teilung des spanischen Erbes aus den Jahren 1698 bis 1700, die englische Thronfolge-Akte von 1701 und die pragmatische Sanktion von 1713 aufgenommen. Abschnitt VI enthält wichtige Dokumente zur Anerkennung neuer Staaten, neuer Herrscher und Regierungen. Dabei ist die Auswahl der wiedergegebenen Dokumente gut gelungen. In Abschnitt VII sind mit dem Cromwell-Manifest zur Eröffnung des Krieges gegen Spanien von 1655, den spanisch-englischen "Amerika-Verträgen" von 1667-1670, den spanisch-portugiesischen Verträgen über die Grenzen in Amerika von 1750 bis 1777 , den Geheiminstruktionen des britischen Königs Georg III für James Cook von 1768 und der britisch-spanischen Nootka Sound Convention von 1790 die wichtigsten Dokumente 
zur Auflösung des spanisch-portugiesischen Kolonisationsmonopols wiedergegeben. Dokumente zu Repressalien und Kaperei, Regeln der Seekriegsführung, Neutralität, Wiedergeburt der Schiedsgerichtsbarkeit, Handelskompanien, und dem Sklavenhandel enthalten die Abschnitte VIII bis XIII. Abschnitt XIV faßt die Verträge zu den Teilungen Polens in den Jahren 1772/73 bzw. 1793 und 1794/95 zusammen. Die völkerrechtlichen Ideen der französischen Revolution werden in Abschnitt XV anhand einer gelungenen Auswahl dokumentiert. Abschnitt XVI trägt den Titel "Europa unter dem Ansturm des revolutionären und des napoleonischen Frankreichs". In diesem Abschnitt sind sowohl die wichtigen Friedensverträge, wie etwa der französisch-preussische Friedensvertrag vom 5. April 1795 oder der österreichisch-französische Friedensvertrag vom 9. Februar 1801 bzw. der französisch-russische Friedensvertrag von 1807 als auch andere bedeutsame völkerrechtliche Dokumente, wie etwa die Rheinbund-Akte vom 12. Juli 1806, die Erklärung Kaiser Franz II uber die Niederlegung der römisch-deutschen Kaiserkrone vom 6. August 1806 und die Achterklärung gegen Napoleon vom 13. März 1815 enthalten. Überraschend ist die Aufnahme der Dokumente zum 2. britisch-"amerikanischen" Krieg von 1812-1814 in diesen Abschnitt.

Insgesamt handelt es sich bei der Quellensammlung von Grewe um ein rundum gelungenes Werk. Dies kann schon anhand der Durchsicht des Bandes 2 festgestellt werden. Sicherlich wird man an der einen oder anderen Stelle der Dokumente streiten können, wie dies im Zusammenhang mit den Verträgen zwischen europäischen und den nicht-christlichen Mächten in Nordamerika angedeutet wurde. Insgesamt ist aber die Auswahl des umfangreichen Materials hervorragend gelungen. Daher werden die "Quellen zur Geschichte des Völkerrechts" ein unverzichtbares Arbeitsmittel für diejenigen darstellen, die sich mit der Völkerrechtsgeschichte beschäftigen.

Dieter Dörr

Ernst-Ulrich Petersmann / Meinhard Hilf (eds.)

The New GATT Round of Multilateral Trade Negotiations - Legal and Economic Problems

Kluwer Publishers, Deventer 1988, 597 p., $£ 144.00$

In September 1986 the GATT Unuguay Round was launched by the Ministerial Declaration of Punta del Este. This new GATT Round of Multilateral Trade Negotiations is intended to reestablish the authority of GATT to extend its rules to new areas of law. However, the General Agreement on Tariffs and Trade is facing serious problems which might endanger the success of the negotations. Though now enjoying a membership of more than 100 countries, the GATT has to cope with less interest in its legal and economic values which 